\title{
Research on Wireless Sensor Networks Topology Models
}

\author{
Ziqing Zhang, Hai Zhao, Jian Zhu, Dazhou Li
}

Information Science and Engineering, Northeastern University, Shenyang, China.

Email: zhangziqing100@163.com, zhhai@neuera.com

Received November $17^{\text {th }}, 2010$; revised November $28^{\text {th }}, 2010$; accepted December $14^{\text {th }}, 2010$.

\begin{abstract}
The topology control is one of the research focuses in wireless sensor networks. Different network topologies will have different effects on the properties of the network, such as the reliability, energy consumption and latency. In order to study the relationship between the topology and the network performance, in this paper we have designed three kinds of topology models which are regular hexagon topology model, plane grid topology model and equilateral triangle topology model. Then the simulation experiments about these models have been implemented in NS2 and the simulation results show that the plane grid topology has the best reliability.
\end{abstract}

Keywords: Wireless Sensor Network, Topology, Network Property, NS2

\section{Introduction}

Wireless sensor networks (WSNs) have wide fields of application [1-2]. As a basic problem in the wireless sensor network, the topology will directly affect the service quality of "perception" which can be provided by the sensor network and the entire network performance. A good network topology has momentous significance for the distribution of network space resources, perception of the environment, information acquisition and the improvement of network viability [3].

Static network topology model is the most basic questions [4]. Only the sensor nodes are disposed well in the destination area can the other work and optimization be further carried on. This article discusses the two-dimensional topology model in the initial deployment of sensor networks [5].

Generally speaking, the initial deployment of nodes mainly includes two ways. deterministic deployment and stochastic deployment [6]. But the stochastic deployment way is very difficult for the numerous sensor nodes to lay aside in suitable position. It's very easy to cause the sensor network covering unreasonable. For example, the sensor nodes have been distributed dense or sparsely in the partial target sector which seriously influence the performance of network. Thereby, the determinism deployment has been mainly discussed in this article. The sensor nodes are deployed in the certain position to cover the number of nodes is requested few as far as possible. The fixed surveillance area completely. Simultaneously. In recent years, the scholars all over the world have conducted relevant problems of topology, and certain progress has been made. Besides, some optimal topological models have been put forward. Among which, there are regular hexagon topology model [7], plane grid topology model [8-12] and equilateral triangle topology model [13] which is the most classical topological model. This paper discusses the three topological models and network performance.

\section{The Topology Models of Sensor Node}

Uniting the application characteristic and the system performance of wireless sensor network [14], when deploying the nodes the factors which should be mainly considered include coverage [15], connection and energy conservation [16]. The supposition of sensor's correspondence radius is equal to $\mathrm{R}$ and all the sensor nodes have similar sensation radius $\mathrm{R}$.

\subsection{Regular Hexagon Topology Model}

The region of interest is divided into a series of closely adjacent grid of regular hexagon.

\subsection{Plane Grid Topology Model}

The region of interest is divided into a series of closely adjacent grid of regular quadrangle. 


\subsection{Equilateral Triangle Topology Model}

The region of interest is divided into a series of closely adjacent grid of equilateral triangle.

The detection area can be covered completely with these three kinds of sensor network and the whole network can be kept working. The node in hexagon topological structure has three neighbor nodes at most, in plane grid topology has four and in equilateral triangle topology there are six.

When the covered area is $1000 \mathrm{~m} \times 1000 \mathrm{~m}$ and the length of topology is fixed $150 \mathrm{~m}$. As can be seen from Figure 1, the hexagon topology network needs 40 sensor nodes, the plane grid topology needs 49 sensor nodes and equilateral triangle topology needs 56 sensor nodes. This means that, when the same size of region is covered with the same quantity of sensor nodes. The length of topology arranged in hexagon is the shortest, second is the plane grid structure and the longest is the equilateral triangle topology.

If in the network each node's wireless signal covers massive other nodes, this will cause collision frequently which affect the correspondence between the nodes, reduce the turnover rate and increase network delay.

\section{The Result of the Experiment}

The experiment will be carried in simulation platform NS2. The three kinds of topological models will be simulated and their performances will be analyzed. The parameters are established as in Table 1. The performance indicators which will be test are showed in Table 2 .

\subsection{The Experiment of Hexagon Topology}

After arranging the nodes which can be seen from Figure 2(a), the length of topology is $130 \mathrm{~m}$. Each node in the network communicates with No. 24 node in the center. The Figure 2(b) shows the charge ratio, Figure 2(c) shows the time delay and Figure 2(d) shows the energy consumption in entire network. The data in No. 0 node expresses that No. 0 node as the source node transmits 100 data packets to No. 24 goal node altogether. The charge ratio is 0.30 .

The data in No. 13 node expresses that No. 13 node as the source node transmits 100 data packets to No. 24 goal node altogether. The charge ratio is 0.65 .

\subsection{The Experiment of Plane Grid Topology}

After arranging the nodes which can be seen from Figure 3(a), the length of topology is $140 \mathrm{~m}$.

The length of topology in Figure $\mathbf{2}$ is $130 \mathrm{~m}$ and in Figure 3 is $140 \mathrm{~m}$. This means that when the same quantity of sensor nodes are arranged in the same size of region, the hexagon structure will be denser.
The ratio of reception in Figure 3(b) is higher than that of Figure 2(b). Because the node in the sensor network formed plane grid topology structure has more neighbor nodes. The possible way for the data message arriving at the goal node will be more. Therefore the rate of receiving package is higher.

A comparison of Figure 2(c) and Figure 3(c) indicates that the time delay in Figure 3(c) is longer. In Figure 2(c), the time delay is $0.0350 \mathrm{~s}$ when the data packet is transmitted from No. 20 node to No. 24 node.

In Figure 3(c), the time delay is $0.0437 \mathrm{~s}$ which is greater than $0.0350 \mathrm{~s}$. Because each node has more neighbor nodes, not all the data packets arrive at the goal node along the shortest path from the source node. So the delay is longer. In addition, the communication nodes between the source node and the goal node are more. Wireless signals will conflict frequently when transmitting the data message. So the network delay is increased.

Consumption is more in Figure 3(d) than that of Figure 2(d). Because in the network formed plane grid

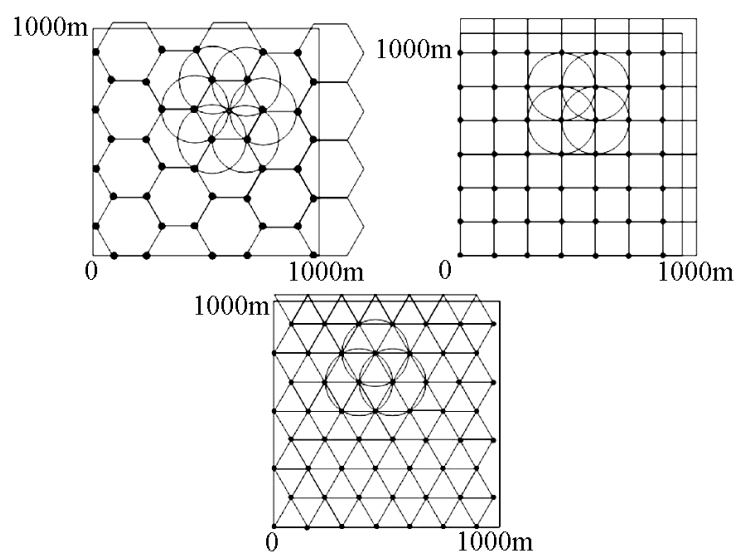

Figure 1. Three kinds of typical topology models.

Table 1. The establishment of parameter.

\begin{tabular}{cc}
\hline The scene will be simulated & $1000 \mathrm{~m}^{*} 1000 \mathrm{~m}$ \\
\hline The number of nodes & 50 \\
Wireless transmission model & Shadowing \\
TTL & 13 \\
The distance of communica- & Charge ratio $\mathrm{p}=0.34$ \\
tion is 150 & Flooding \\
Routing protocol & 100 data packets, the \\
The data packets sent to the & goal node
\end{tabular}

Table 2. The performance indicators will be test for the network.

\begin{tabular}{ll}
\hline 1 & the ratio of reception \\
\hline 2 & time delay \\
3 & energy consumption in entire network \\
\hline
\end{tabular}




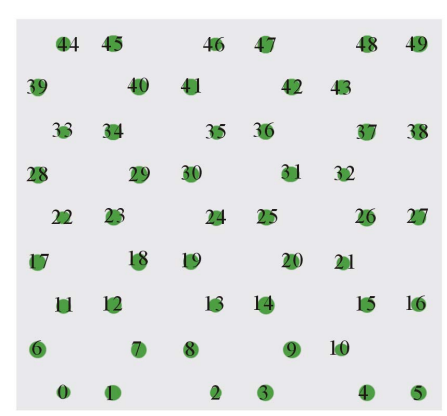

(a)

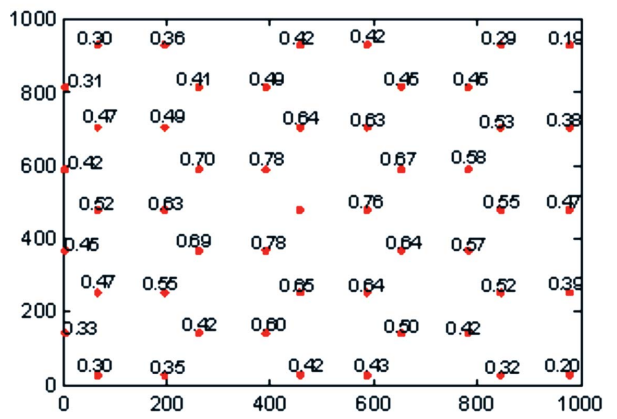

(b)

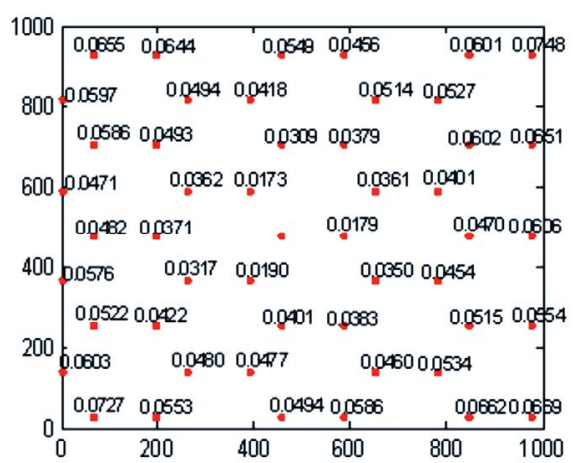

(c)

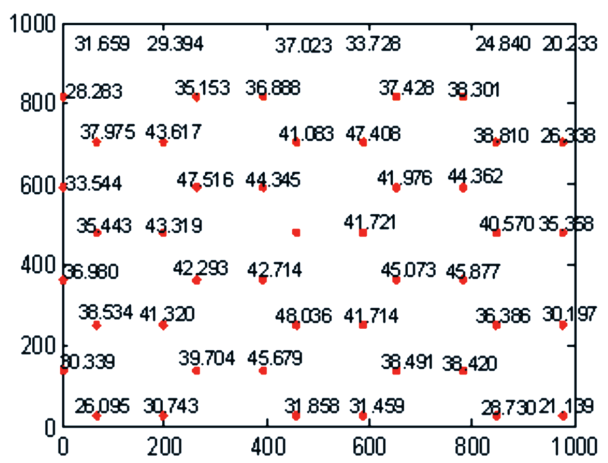

(d)

Figure 2. Regular hexagon topology model.

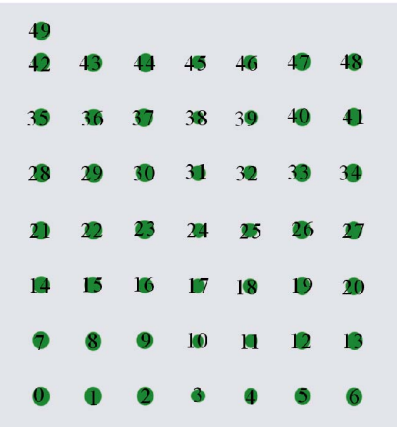

(a)

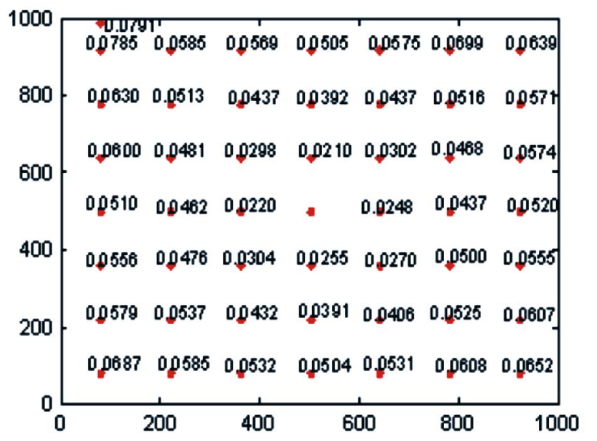

(c)

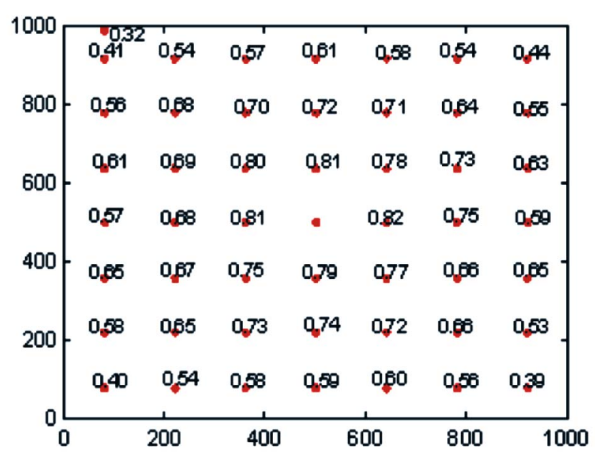

(b)

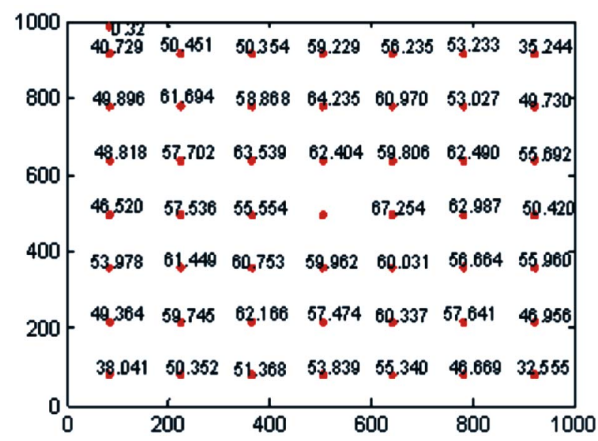

(d)

Figure 3. Planar grid topology model. 


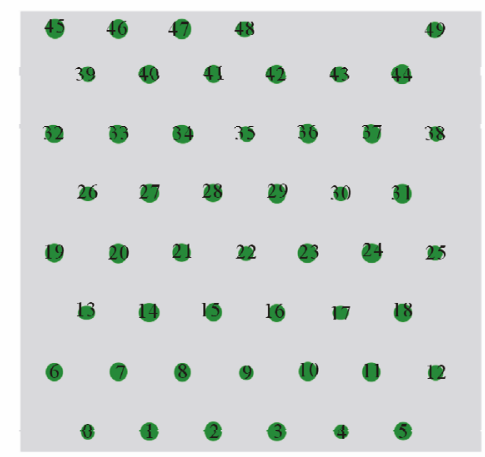

(a)

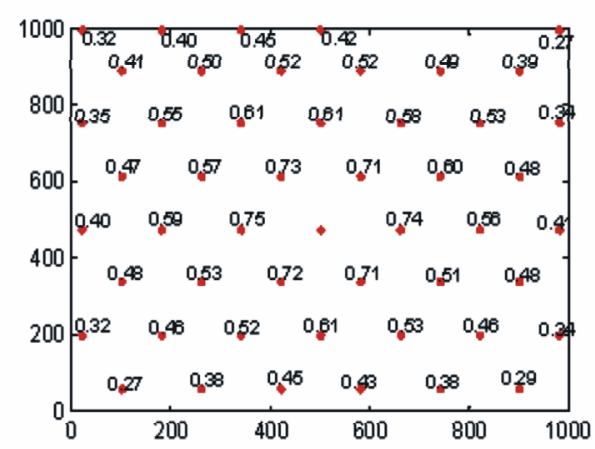

(b)

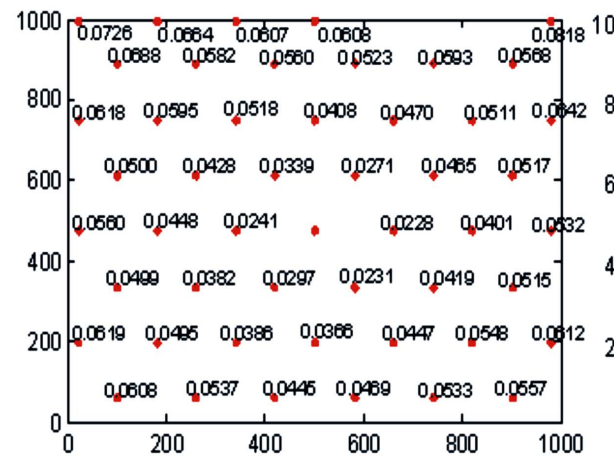

(c)

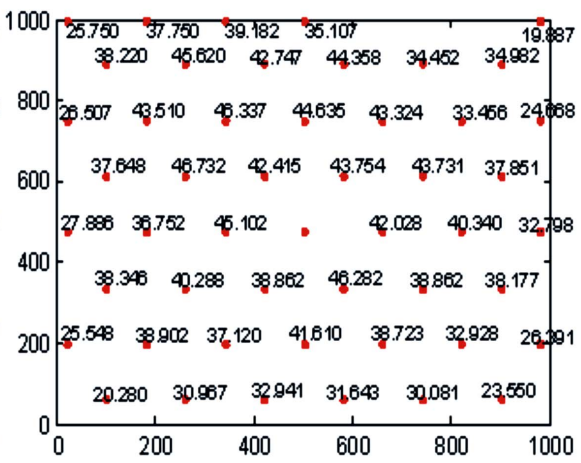

(d)

Figure 4. Equilateral triangle topology model.

structure the node will receive more of the packets. The intermediate nodes will consume more energy to transmit and receive packets. So the total energy consumption of network is more.

\subsection{The Experiment of Equilateral Triangle Topology}

After arranging the nodes which can be seen from Figure 4(a), the length of topology is $160 \mathrm{~m}$.

The length of topology in Figure $\mathbf{2}$ is $\mathbf{1 3 0} \mathrm{m}$, in Figure 3 is $140 \mathrm{~m}$ and in Figure 4 is $160 \mathrm{~m}$. This means that when the same quantity of sensor nodes are arranged in the same size of region, the equilateral triangle structure will be sparser.

The comparison between Figure 3(b) and Figure 4(b) indicates that the rate of receiving package is higher in the network formed plane grid structure. Although the node has less neighbor nodes in the plane grid topology sensor network, the length of topology is shorter in this kind of network. So the rate of receiving package is higher. Furthermore, in the equilateral triangle topology network each node has more neighbor nodes which induce collision for the wireless signal and affect the reception of the data packet.

\section{Conclusions}

In this paper three kinds of wireless sensor network topology models are proposed which have been simulated separately on simulation platform NS2. After the analysis we can see that the distance between the source node and goal node and the quantity of the neighbor nodes affect the charge ratio, delay and the network energy consumption. The simulation experiments show that when the same quantity of sensor nodes are arranged in the same size of region, the length of topology in the hexagon structure is the shortest, second is plane grid topology model and in the equilateral triangle topology network the length of topology is the longest. The rate of receiving package is the highest in the plane grid topology sensor network, second is the hexagon structure and the lowest is equilateral triangle topology network. Finally through a series of comparison we can see that the network reliability of plane grid topology model is the best.

\section{REFERENCES}

[1] I. F. Akyildiz, W. Su, Y. Sankarasubramaniam and E. Cayirci, "Wireless Sensor Networks: A Survey," Computer Networks, Vol. 38, No. 4, 2002, pp. 393-422. doi: 
10.1016/S1389-1286(01)00302-4

[2] S. Tilak, N. B. Abu-Ghazaleh and W. Heinzelman, "A Taxonomy of Wireless Microsensor Network Models," ACM Mobile Computing and Communications Review, Vol. 6 No. 2, 2002, pp. 28-36. doi:10.1145/565702. 565708

[3] C. F. Huang and Y. C. Tseng, "The Coverage Problem in a Wireless Sensor Network," Proceedings of ACM WSNA, San Diego, 19 September 2003, pp.519-528 doi:10.1145/ 941350.941367

[4] C. Shen, C. Srisathapornphat and C. Jaikaeo, "Sensor Information Networking Architecture and Applications,” IEEE Personal. Communication, Vol. 8, No. 4, 2001, pp. 52-59. doi:10.1109/98.944004.

[5] S. H. Yang, M. L. Li, and J. Wu, "Scan-Based Movement-Assisted Sensor DePloyment Methodin Wireless Sensor Networks," IEEE Transactions on Parallel and Distributed Systems, Vol. 18, No. 8, 2007, pp.1108-1121. doi:10.1109/TPDS.2007.1048

[6] A. Salhieh, J. Weinmann, M. Kochhal and L. Schwiebert, "Power Efficient Topologies for Wireless Sensor Networks,” International Conference on Parallel Processing, Valencia, 3-7 Sepmber 2001, pp. 156-163.

[7] K. Chakrabarty, S. S. Iyengar, H. R. Qi and E. C. Cho., "Grid Coverage for Surveillance and Target Location in Distributed Sensor Networks," IEEE Transactions on Computers. Vol. 51, No. 12, 2002, pp. 1448-1453. doi: 10.1109/TC.2002.1146711

[8] Q. S. Wu, N. S. V. Rao and X. J. Du, et al., "On Efficient Deployment of Sensors on Planar Grid," IEEE Computer Communications. Vol. 30, No. 14-15, 2007, pp. 2721-2734. doi:10.1016/j.comcom.2007.05.012

[9] K. Chakrabarty, S. S. Iyengar, H. R. Qi and E. Cho, “Grid
Coverage for Surveillance and Target Location in Distributed Sensor Networks," IEEE Transactions on Computers, Vol. 51, No. 12, 2002, pp. 1448-1453. doi: 10.1109/TC.2002.1146711

[10] S. Shakkottai, R. Srikant and N. Shroff. "Unreliable Sensor Grids: Coverage, Connectivity and Diameter." IEEE Press, San Francisco, 2003.

[11] C.-H. Wu, K.-C. Lee and Y.-C. Chung, “A Delaunay Triangulation Based Method for Wireless Sensor Network Deployment," IEEE Computer Communications, Vol. 30, No. 14-15, 2007, pp. 2744-2752. doi:10.1016 /j.comcom.2007.05.017

[12] J. L. Hill, "System Architecture for Wireless Sensor Networks,” Ph.D. Thesis, Computer Science University of California , Berkeley, 2003.

[13] Q. Jiang, D. Manivannan, "Routing protocols for sensor networks," Consumer Communications and Networking Conference, Las Vegas, 5-8 January 2004, pp. 93-98.

[14] S. S. Dhillon and K. Chakrabarty, "Sensor Placement for Effective Coverage and Surveillance in Distributed Sensor Networks," IEEE Wireless Communications and Networking Conference, New Orleans, 20-20 March 2003, pp. 1609-1614.

[15] S. Meguerdichian, F. Koushanfar, M. Potkonjak and M. Srivastava, "Coverage Problems in Wireless Ad Hoc sensor Networks,” Proceedings of IEEE Infocom, Alaska, 22-26 April 2001, pp. 22-26.

[16] Y. C. Wang, C. C. Hu and Y. C. Tseng, "Efficient Deployment Algorithms for Ensuring Coverage and Connectivity of Wireless Sensor Networks," Wireless Internet Conference, Budapest, 10-14 July 2005, pp. 114-121. doi:10.1109/WICON.2005.13 